\title{
Application of Fast Multipole Methods to the NASA Fast Scattering Code
}

\author{
Mark H. Dunn ${ }^{*}$ \\ Ana F. Tinetti* \\ Yorktown, VA 23693
}

The NASA Fast Scattering Code (FSC) is a versatile noise prediction program designed to conduct aeroacoustic noise reduction studies. The equivalent source method is used to solve an exterior Helmholtz boundary value problem with an impedance type boundary condition. The solution process in FSC v2.0 requires direct manipulation of a large, dense system of linear equations, limiting the applicability of the code to small scales and/or moderate excitation frequencies. Recent advances in the use of Fast Multipole Methods (FMM) for solving scattering problems, coupled with sparse linear algebra techniques, suggest that a substantial reduction in computer resource utilization over conventional solution approaches can be obtained. Implementation of the single level FMM (SLFMM) and a variant of the Conjugate Gradient Method (CGM) into the FSC is discussed in this paper. The culmination of this effort, FSC v3.0, was used to generate solutions for three configurations of interest. Benchmarking against previously obtained simulations indicate that a twenty-fold reduction in computational memory and up to a four-fold reduction in computer time have been achieved on a single processor.

\section{Nomenclature}

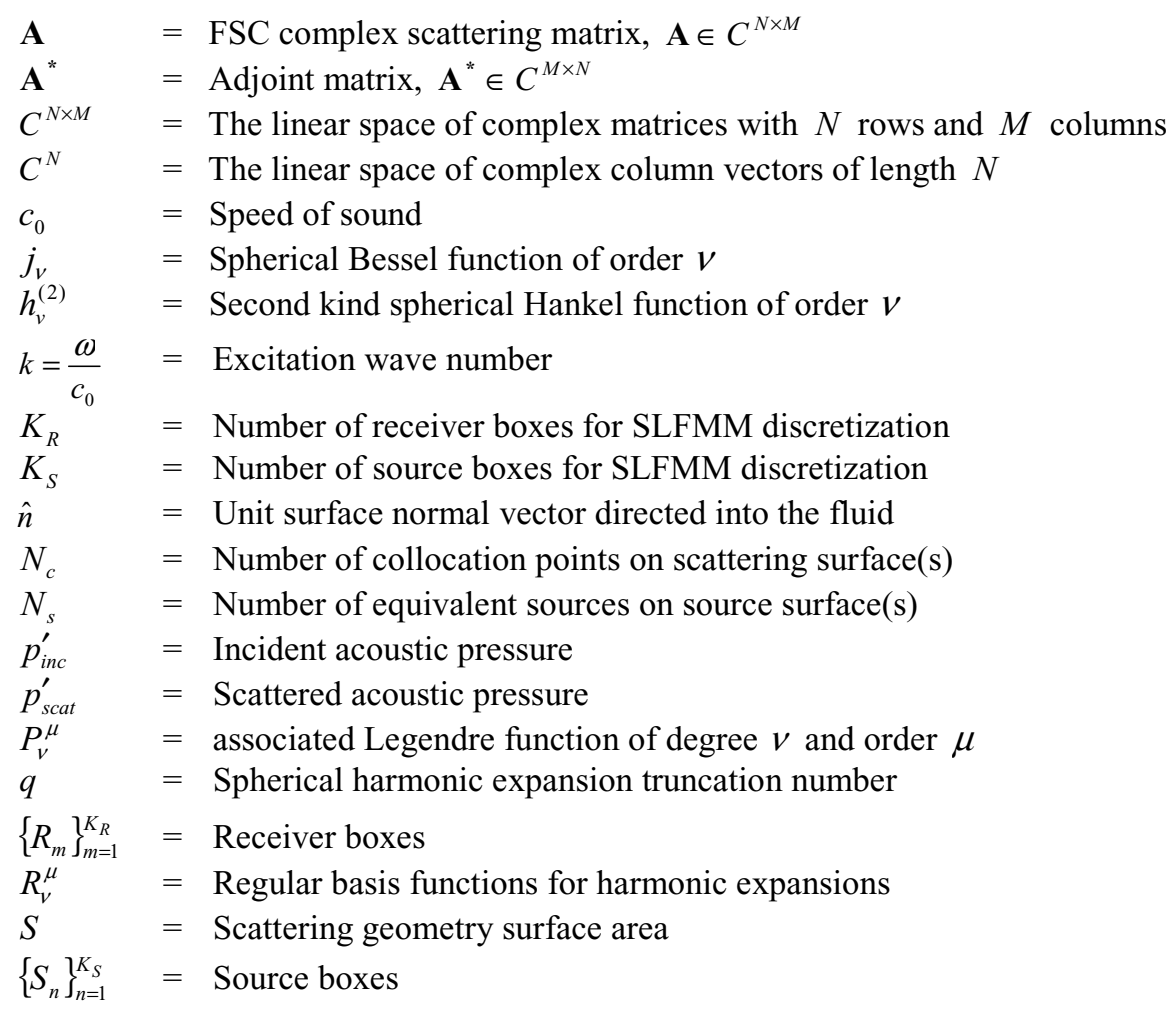

\footnotetext{
${ }^{*}$ Consultant, Senior Member AIAA
} 


$$
\begin{array}{ll}
\left\{S_{n}^{-}\right\}_{n=1}^{K_{S}} & =\text { Source box enveloping sphere interiors } \\
\left\{S_{n}^{+}\right\}_{n=1}^{K_{S}} & =\text { Source box enveloping sphere exteriors } \\
S_{v}^{\mu} & =\text { Singular basis functions for harmonic expansions } \\
\vec{u}_{i n c}^{\prime} & =\text { Incident acoustic velocity } \\
\vec{u}_{s c a t}^{\prime} & =\text { Scattered acoustic velocity } \\
(\vec{x}, t) & =\text { Space-time coordinates } \\
\left\{\vec{x}_{m}\right\}_{m=1}^{N_{c}} & =\text { Collocation points on scattering surfaces } \\
\left\{\vec{x}_{m}^{*}\right\}_{m=1}^{K_{R}} & =\text { Receiver box centers } \\
\left\{\vec{y}_{n}\right\}_{n=1}^{N_{c}} & =\text { Source points on source surfaces } \\
\left\{\vec{y}_{n}^{*}\right\}_{n=1}^{K_{S}} & =\text { Source box centers } \\
Y_{v}^{\mu} & =\text { Spherical harmonics } \\
\alpha & =\text { Surface normal admittance } \\
(\rho, \theta, \varphi) & =\text { Spherical coordinates } \\
\rho_{0} & =\text { Fluid density } \\
\omega & =\text { Excitation frequency }
\end{array}
$$

\section{Introduction}

Successful aircraft deployment requires an accurate assessment of its impact on community noise. Preceding any experimental study of noise abatement techniques, engineers rely on physically accurate computational methods to reduce the set of parameters to be investigated. The NASA Fast Scattering Code (FSC), developed at the Langley Research Center (LaRC), is a versatile noise prediction program for evaluating noise reduction concepts such as the shielding effects of scattering geometry and engine nacelle placement, or sound absorption through liner optimization. The code calculates the time harmonic acoustic field produced by the interaction of known incident sound with aero-surfaces, of arbitrary shape and surface impedance, immersed in a potential flow. Researchers have applied the FSC to a variety of aeroacoustic simulations involving fixed-wing and rotary-wing configurations, as detailed in references $1-5$.

The FSC uses the equivalent source method (ESM) to solve an exterior three-dimensional Helmholtz boundary value problem (BVP) by expanding the scattered acoustic pressure field into a series of point monopoles or dipoles. The point sources are located on a fictitious surface located interior to the actual scattering surface. Users input surface geometry, background fluid properties, incident fields for both acoustic pressure and velocity, and operating conditions. The code then calculates the scattered acoustic pressure field at user specified locations in space and time. Details on the solution methodology and program usage are given in references 6 and 7.

The use of boundary techniques - such as the ESM, finite element methods, boundary integral methods, or boundary element methods - for solving BVPs involves discretization of the scattering surfaces and the solution of a large (usually) dense system of linear equations. For the ESM, an over-determined linear system is produced where the number of unknown equivalent monopole source strengths is approximately equal to the product of scattering surface area $(S)$ and the square of the incident sound excitation wave number $(k)$ :

$$
N_{s} \approx S k^{2}
$$

As implemented in the FSC, the ESM requires approximately one-third the memory of the other BVP solution methods and about one-ninth the computational time (accounting for the adjective Fast in FSC). Even with these computational gains, FSC version 2.0 predictions using current single-processor workstation technology with less than $30 \mathrm{~GB}$ of shared memory are limited to conditions where the number of equivalent sources $\left(N_{s}\right)$ does not exceed about 25000. Simulations for high frequency/large scattering surface combinations, such as a full scale commercial transport, yield large linear systems with considerably more unknowns that can only be solved with advanced computer systems or by employing numerical algorithms that drastically reduce computational resource utilization. 
Recent advances in the use of Fast Multipole Methods (FMM) for solving scattering problems coupled with sparse linear algebra techniques, suggest a substantial reduction in the consumption of computer resources relative to dense matrix based boundary methods. ${ }^{8}$. Implementation of FMM into the FSC is described in the present paper. In the resulting computer program, FSC v3.0, the dense, linear least squares solution approach of FSC v2.0 is replaced with an iterative conjugate gradient method (CGM) that features FMM based fast field calculations and fast matrix-vector multiplication algorithms. Numerical scattering studies are presented that show an order-of-magnitude reduction in computer memory and computational time. This results in the ability to quadruple the number of unknowns in the linear system to be solved, which equates to doubling the maximum frequency achievable with FSC v2.0 for a given problem. Discussions in the remainder of the paper are grouped into two categories: 1) FSC computational advances, and 2) aeroacoustic applications including high frequency numerical simulations for full scale nacelle and commercial transport configurations.

\section{FSC Computational Advances}

Technical aspects of fast multipole methods as they pertain to the FSC version 3.0 are discussed in this section. First, the Single-Level Fast Multipole Method (SLFMM) is introduced for the rapid calculation of the scattered field produced by a collection of monopoles. Next, memory reduction algorithms are developed for solving the FSC linear system using conjugate gradient methods in which the SLFMM is used for fast vector-matrix multiplications at each iteration of the CGM. Much of the terminology and notation used for the FMM analysis presented here was taken from reference 8. This comprehensive text contains detailed analyses of the FMM concepts and a wealth of references that follow the historical developments of the subject and its research applications.

\section{A. Single Level Fast Multipole Method}

For the monopole variant of the ESM, the scattered acoustic pressure at any spatial location $\vec{x}$ is written as a point monopole superposition ( $e^{+i \omega t}$ time dependence is assumed):

$$
p_{s c a t}^{\prime}(\vec{x})=\sum_{m=1}^{N_{S}} c_{m} \frac{e^{-i k\left|\vec{x}-\vec{y}_{m}\right|}}{4 \pi\left|\vec{x}-\vec{y}_{m}\right|}
$$

In Eq. (2), $\left\{\vec{y}_{m}\right\}_{m=1}^{N_{s}}$ are the spatial locations of the source points and the complex numbers $\left\{c_{m}\right\}_{m=1}^{N_{s}}$ are the source strengths. The source points are distributed inside the scattering geometry and the expansion strengths are tuned to satisfy a general impedance type boundary condition at the $N_{c}$ distinct collocation points $\left\{\vec{x}_{n}\right\}_{n=1}^{N_{c}}$ that lie on the scattering surfaces. For FSC applications $N_{c} \approx 3 N_{s}$. The resulting over-determined linear system is solved using least squares methods as discussed in the next sub-section.

As it pertains to the ESM, the basic concept of the FMM is to replace the field due to a single point monopole located at $\vec{y}$ by an infinite series of spherical harmonics in which the source point and field (or receiver) point are separated. The fundamental identity of the process is given by Eq. (3) and can be derived by applying the method of separation of variables to the three-dimensional Helmholtz differential equation in spherical coordinates.

$$
\frac{e^{-i k|\vec{x}-\vec{y}|}}{4 \pi|\vec{x}-\vec{y}|}= \begin{cases}-i k \sum_{v=0}^{\infty} \sum_{\mu=-v}^{v} S_{v}^{-\mu}(\vec{y}) R_{v}^{\mu}(\vec{x}) & |\vec{x}|<|\vec{y}| \\ -i k \sum_{v=0}^{\infty} \sum_{\mu=-v}^{v} R_{v}^{-\mu}(\vec{y}) S_{v}^{\mu}(\vec{x}) & |\vec{y}|<|\vec{x}|\end{cases}
$$

$\operatorname{In}(3)$,

$$
\begin{gathered}
R_{v}^{\mu}(\vec{\xi})=j_{v}(k \rho) Y_{v}^{\mu}(\theta, \varphi) \\
S_{v}^{\mu}(\vec{\xi})=h_{v}^{(2)}(k \rho) Y_{v}^{\mu}(\theta, \varphi)
\end{gathered}
$$

are referred to as regular and singular basis functions, respectively, where $(\rho, \theta, \varphi)$ are spherical coordinates corresponding to the Cartesian coordinates $\vec{\xi}, j_{v}$ is the first kind spherical Bessel function of order $v, h_{v}^{(2)}$ is the second kind spherical Hankel function of order $v$, and the spherical harmonics $Y_{v}^{\mu}$ are given by the formulas 


$$
Y_{v}^{\mu}(\theta, \varphi)=(-1)^{\mu} \sqrt{\frac{2 v+1}{4 \pi} \frac{(v-|\mu|) !}{(v+|\mu|) !}} P_{v}^{|\mu|}(\cos \varphi) e^{i \mu \theta}
$$

with $P_{v}^{|\mu|}$ denoting the associated Legendre function of degree $v$ and order $|\mu|$. The singular and regular terminology refers to the behavior of the spherical Bessel functions at $\rho=0$. Fast multipole algorithms feature truncated spherical harmonic expansions of both the singular and regular type for the approximation of the scattered acoustic field of equation (2). Direct evaluation of Eq. (2) at $N$ receiver points requires $O\left(N N_{s}\right)$ mathematical operations. By achieving a large degree of source-receiver point separation, the FMM can significantly reduce the computational complexity of field calculations as we now demonstrate.

Suppose all source points lie inside some sphere of finite radius. Let $B^{+}$and $B^{-}$denote the exterior and interior regions of the sphere, respectively; then combining Eqs. (2) and (3) and formally interchanging the order of summation yields the so-called middleman algorithm (MA) for calculating the scattered field:

$$
p_{s c a t}^{\prime}(\vec{x})= \begin{cases}\sum_{v=0}^{\infty} \sum_{\mu=-v}^{v} C_{v}^{\mu} R_{v}^{\mu}(\vec{x}) & \vec{x} \in B^{-} \\ \sum_{v=0}^{\infty} \sum_{\mu=-v}^{v} D_{v}^{\mu} S_{v}^{\mu}(\vec{x}) & \vec{x} \in B^{+}\end{cases}
$$

Where

$$
\begin{aligned}
& C_{v}^{\mu}=-i k \sum_{m=0}^{N_{s}} c_{m} S_{v}^{-\mu}\left(\vec{y}_{m}\right) \\
& D_{v}^{\mu}=-i k \sum_{m=0}^{N_{s}} c_{m} R_{v}^{-\mu}\left(\vec{y}_{m}\right)
\end{aligned}
$$

If for some prescribed truncation error tolerance $\varepsilon$, the expansions in Eq. (6) converge after the first $v=q-1$ terms, then the number of mathematical operations required to evaluate Eq. (6) at $N$ receiver points is $O\left(q^{2} N\right)$ and $O\left(q^{2} N_{s}\right)$ operations to compute Eq. (7). Furthermore, if $q^{2} \ll N_{s}$, then the total mathematical complexity is $O\left(N+N_{s}\right)$ compared to $O\left(N N_{s}\right)$ for direct evaluation using Eq. (2). The computational gains are entirely attributable to the separation of source and receiver interaction.

For general scattering problems, the middleman algorithm fails because the sphere that envelops all sources is too large for the rapid convergence of Eq. (6). The SLFMM algorithm applies the MA to smaller spatial regions and has been implemented into the FSC. The process begins with the discretization of the computational domain into cubical boxes and the partitioning of source and receiver points into the boxes. The box size depends on the excitation frequency and size of the computational domain. Note that some boxes may contain both source and receiver points. Figure 1 shows the partitioning scheme for a futuristic large-scale commercial transport with three engine nacelles (SAX-40) ${ }^{9}$. Point monopoles are distributed over the entire source surface which lies slightly interior to the actual scattering surface. For this demonstration, it is desired to compute the scattered acoustic field on a plane of discrete observer locations bisecting the airframe as shown in the figure. 


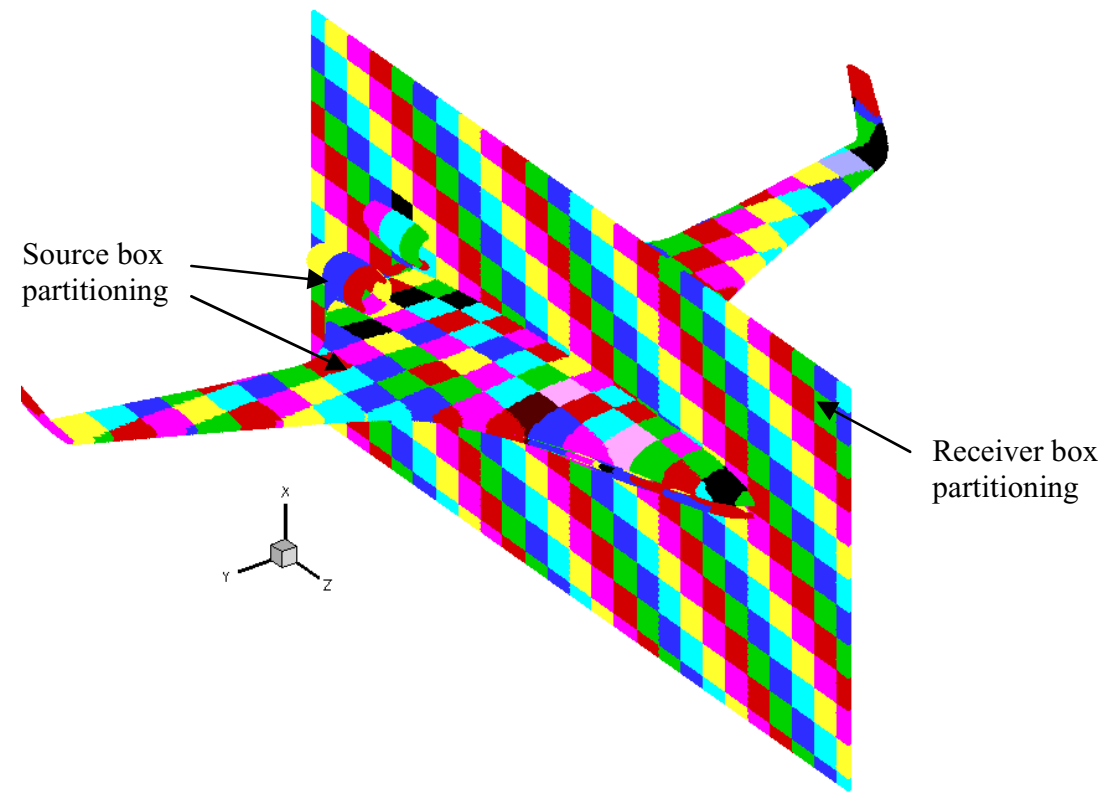

Figure 1. Source and receiver point partitioning for the $S A X-40$ configuration, $f=400 \mathrm{~Hz}$.

Let $\left\{S_{n}\right\}_{n=1}^{K_{S}}$ denote the collection of non-empty source boxes with box centers $\left\{\vec{y}_{n}^{*}\right\}_{n=1}^{K_{S}}$, and $\left\{R_{m}\right\}_{m=1}^{K_{R}}$ the nonempty receiver boxes with centers $\left\{\vec{x}_{m}^{*}\right\}_{m=1}^{K_{R}}$. We use the notation $S_{n}^{+}$and $S_{n}^{-}$for the exterior and interior, respectively, of the smallest sphere that totally contains the $n$-th source box. A similar notation is used for the receiver box array.

The radius of the sphere is given by $d=\frac{\sqrt{3}}{2} d_{\text {cube }}$, where $d_{\text {cube }}$ is the length of the side of a cube. The term wellseparated is used to describe two boxes whose centers are greater than $2 d$ apart. The superposition given by Eq. (2) is distributed across the $K_{S}$ source boxes

$$
p_{s c a t}^{\prime}(\vec{x})=\sum_{n=1}^{K_{S}} p_{n}^{\prime}(\vec{x})=\sum_{n=1}^{K_{S}} \sum_{\vec{y}_{\alpha} \in S_{n}} c_{m} \frac{e^{-i k\left|\vec{x}-\vec{y}_{\alpha}\right|}}{4 \pi\left|\vec{x}-\vec{y}_{\alpha}\right|}
$$

and evaluated according to the SLFMM algorithm as follows:

Step 1) For $n=1, \ldots, K_{S}$, compute singular expansion coefficients for source box $n$ :

$$
D_{v}^{\mu}=-i k \sum_{\vec{y}_{\alpha} \in S_{n}} c_{\alpha} R_{v}^{-\mu}\left(\vec{y}_{\alpha}-\vec{y}_{n}^{*}\right)
$$

Step 2) For each receiver box $R_{m}, m=1, \ldots, K_{R}$ calculate $\vec{t}_{n m}=\vec{x}_{m}^{*}-\vec{y}_{n}^{*}$ and determine if source and receiver boxes are well-separated. If $\left|\vec{t}_{n m}\right|>2 d$, then the boxes are well-separated and the regular expansion is used to compute the field for each point in the receiver box:

$$
p_{n}^{\prime}(\vec{x})=\sum_{v=0}^{q-1} \sum_{\mu=-v}^{v} C_{v}^{\mu} R_{v}^{\mu}\left(\vec{x}-\vec{x}_{m}^{*}\right)
$$

Where

$$
C_{v}^{\mu}=-i k \sum_{\vec{y}_{\alpha} \in S_{n}} c_{\alpha} S_{v}^{-\mu}\left(\vec{y}_{\alpha}-\vec{x}_{m}^{*}\right)
$$


If $\left|\vec{t}_{n m}\right| \leq 2 d$, then source and receiver boxes are not well-separated and the singular expansion is used to calculate the field for receiver points outside the source box sphere, or the direct summation is used for receiver points that lie inside the source box sphere:

$$
p_{n}^{\prime}(\vec{x})=\left\{\begin{array}{l}
\sum_{\vec{y}_{\alpha} \in S_{n}} c_{\alpha} \frac{e^{-i k\left|\vec{x}-\vec{y}_{\alpha}\right|}}{\left|\vec{x}-\vec{y}_{\alpha}\right|} \quad \vec{x} \in S_{n}^{-} \cap R_{m} \\
\sum_{v=0}^{q-1} \sum_{\mu=-v}^{v} D_{v}^{\mu} S_{v}^{\mu}\left(\vec{x}-\vec{y}_{n}^{*}\right) \quad \vec{x} \in S_{n}^{+} \cap R_{m}
\end{array}\right.
$$

For large-scale scattering problems, most source-receiver box combinations are well-separated and the computational cost is dominated by the evaluation of Eqs. (10) and (11). Direct evaluation of the monopole sum in Eq. (12) occurs infrequently, and for some applications considered here accounts for less than $5 \%$ of the computational time. If the number of receiver points is of the same order of magnitude as source points, then analysis shows ${ }^{8}$ that the computational complexity of the SLFMM is $O\left(q^{4 / 3} N_{s}^{4 / 3}\right)$ compared to $O\left(N_{s}^{2}\right)$ for $100 \%$ direct evaluation. For $q^{2}<<N_{s}$ the savings are considerable. Further speed-up is possible by employing fast translation methods for the evaluation of the regular expansion coefficients of Eq. (11) given the singular expansion coefficients of Eq. (9). As discussed in detail in chapters 3, 7, and 8 of reference 8, fast translation algorithms reduce the SLFMM complexity to $O\left(q N_{s}^{4 / 3}\right)$.

The SLFMM algorithm presented in Eqs. (8) through (12) has been implemented into the FSC v3.0 for predicting the scattered acoustic pressure field and its gradient and applied to known solutions from FSC v2.0 for comparison as reported in section III. Pressure gradient calculations require application of the gradient operator to the singular and regular basis functions of Eq. (4). Analytical details of this process are lengthy and the resulting recursion relationships can be found in chapter 2 (section 2) of reference 8. The SLFMM has also been deployed in the FSC v3.0 for fast matrix-vector multiplication during the linear algebra phase of the FSC process, as described below.

\section{B. Linear Algebra Considerations}

The FSC least squares matrix $\mathbf{A} \in C^{N_{c} \times N_{s}}$ is obtained by requiring the expansion given in Eq. (2) to satisfy an impedance type boundary condition at the $N_{c}$ collocation points $\{\vec{x}\}_{m=1}^{N_{c}}$ on the scattering surface(s):

$$
\mathbf{A}_{j m}=\frac{i}{\rho_{0} c_{0} k} \hat{n}_{j} \cdot \nabla g_{j m}+\alpha_{j} g_{j m} \quad j=1, \ldots, N_{c} \quad m=1, \ldots, N_{s} \quad N_{c}>N_{s}
$$

Where

$$
g_{j m}=\frac{e^{-i k R_{j m}}}{4 \pi R_{j m}} \quad R_{j m}=\left|\vec{x}_{j}-\vec{y}_{m}\right|
$$

In Eq. (13), $\hat{n}_{j}$ is the unit outer surface normal and $\alpha_{j}$ denotes the user supplied surface normal admittance at the $j$-th collocation point. Application of the boundary condition to the incident sound yields the complex source vector $\vec{b} \in C^{N_{c}}$.

$$
b_{j}=-\left[\frac{i}{\rho_{0} c_{0} k} \vec{u}_{i n c}^{\prime}\left(\vec{x}_{j}\right) \cdot \hat{n}_{j}+\alpha_{j} p_{i n c}^{\prime}\left(\vec{x}_{j}\right)\right] \quad j=1, \ldots, N_{c}
$$

In the FSC v2.0, the overdetermined linear system

$$
\mathbf{A} \vec{c}=\vec{b}
$$

for the unknown monopole source strengths $\vec{c} \in C^{N_{s}}$ is premultiplied by the complex conjugate matrix (adjoint matrix) $\mathbf{A}^{*} \in C^{N_{s} \times N_{c}}$ to produce the $N_{s} \times N_{s}$ Hermitian system

$$
\mathbf{A}^{*} \mathbf{A} \vec{c}=\mathbf{A}^{*} \vec{b}
$$

6

American Institute of Aeronautics and Astronautics 
which is then solved using standard Gauss elimination techniques. The preconditioning step, Eq. (17), dominates the solution process and requires $O\left(N_{c} N_{s}\right)$ memory and $O\left(N_{c}^{2} N_{s}\right)$ multiplications to complete. For large-scale problems the computational requirements are well beyond the capabilities of 2008 workstation technology.

To reduce the computational complexity of solving Eq. (16), sparse, iterative linear algebra techniques based on the conjugate gradient method have been incorporated in the FSC v3.0. The methodology features SLFMM algorithms for fast matrix-vector multiplication. As a result, the direct matrix-matrix multiply in Eq. (17) is avoided and memory requirements are reduced by $95 \%$ or more for some of the applications considered here.

\section{Conjugate Gradient Algorithm}

There are several variants of the CGM in the literature (see reference 9 for a review). The algorithm presented below was chosen for its compatibility with the FSC/FMM methodology.

Step 1: For initial guess $\vec{c}^{(0)}$ set $\vec{r}^{(0)}=\vec{b}-\mathbf{A} \vec{c}^{(0)}, \quad \vec{p}^{(0)}=\vec{s}^{(0)}=\mathbf{A}^{*} \vec{r}^{(0)}, \quad \gamma_{0}=\left\|\vec{s}^{(0)}\right\|_{2}^{2}$ where $\|\vec{x}\|_{2}^{2}=\sum_{m=1}^{N_{s}}\left|x_{m}\right|^{2}$

Step 2: For $\lambda=0,1,2, \ldots$ and while $\gamma_{\lambda}>\varepsilon$ for some prescribed tolerance $\varepsilon$ compute

a) $\vec{q}^{(\lambda)}=\mathbf{A} \vec{p}^{(\lambda)}$ and $\alpha_{\lambda}=\gamma_{\lambda}\left\|\vec{q}^{(\lambda)}\right\|_{2}^{-2}$

b) $\vec{c}^{(\lambda+1)}=\vec{c}^{(\lambda)}+\alpha_{\lambda} \vec{p}^{(\lambda)}$

c) $\vec{r}^{(\lambda+1)}=\vec{r}^{(\lambda)}-\alpha_{\lambda} \vec{q}^{(\lambda)}$

d) $\vec{s}^{(\lambda+1)}=\mathbf{A}^{*} \vec{r}^{(\lambda+1)}$ and $\gamma_{\lambda+1}=\left\|\vec{s}^{(\lambda+1)}\right\|_{2}^{2}$

e) $\vec{p}^{(\lambda+1)}=\vec{s}^{(\lambda+1)}+\frac{\gamma_{\lambda+1}}{\gamma_{\lambda}} \vec{p}^{(\lambda)}$

Each iteration of the CGM algorithm involves two matrix-vector multiplies. One multiply involves the original FSC matrix and the other its adjoint. Direct evaluation of either product requires $O\left(N_{c} N_{s}\right)$ multiplications and constitutes the bulk of the numerical work for the CGM. In the next two subsections, fast matrix-vector multiply algorithms are presented that incorporate the SLFMM and reduce the computational complexity an order of magnitude.

Fast Matrix-vector Multiply I

$$
\vec{q}=\mathbf{A} \vec{p} \Rightarrow q_{j}=\sum_{m=1}^{N_{s}} \mathbf{A}_{j m} p_{m}=\left(\frac{i}{\rho_{0} c_{0} k} \hat{n}_{j} \cdot \nabla+\alpha_{j}\right) \sum_{m=1}^{N_{s}} p_{m} g_{j m} \quad j=1, \ldots, N_{c}
$$

The sum on the far right in Eq. (18) is exactly of the form of Eq. (2) and therefore can be evaluated by the SLFMM in $O\left(N_{c}^{4 / 3}\right)$ operations. According to Eq. (12), it is noted that only the portion of the scattering matrix involving source-receiver interactions that are in close proximity need to be stored. Additional arrays of size $O\left(q^{2} N_{c}\right)$ are also required for certain spherical harmonic information that is used repeatedly.

Fast Matrix-vector Multiply II

$$
\begin{gathered}
\vec{s}=\mathbf{A}^{*} \vec{r} \Rightarrow \vec{s}^{*}=\vec{r}^{*} \mathbf{A} \\
\vec{s}^{*}=\vec{r}^{*} \mathbf{A} \Rightarrow \bar{s}_{m}=\sum_{j=1}^{N_{c}} \bar{r}_{j} \mathbf{A}_{j m}=\sum_{j=1}^{N_{c}} \bar{r}_{j}\left(\frac{i}{\rho_{0} c_{0} k} \hat{n}_{j} \cdot \nabla g_{j m}+\alpha_{j} g_{j m}\right) \quad m=1, \ldots, N_{s}
\end{gathered}
$$

In Eq. (20), the bar over a variable denotes the complex conjugate. Expanding the gradient operator and rearranging terms yields

$$
\bar{s}_{m}=\sum_{l=1}^{3} \frac{\partial}{\partial x_{l}} \sum_{j=1}^{N_{c}} d_{j}^{l} g_{j m}+\sum_{j=1}^{N_{c}} e_{j} g_{j m} \quad m=1, \ldots, N_{s}
$$

Where 


$$
d_{j}^{l}=\frac{i}{\rho_{0} c_{0} k} \hat{n}_{j}^{l} \bar{r}_{j} \text { and } e_{j}=\alpha_{j} \bar{r}_{j} \quad j=1, \ldots, N_{c} ; \quad l=1,2,3
$$

Each of the sums over the index $j$ in Eq. (21) is amenable to SLFMM calculations. Again, the computational complexity is reduced to $O\left(N_{c}^{4 / 3}\right)$.

\section{Full Scale Applications}

The advantages associated with the computational enhancements implemented into FSC v3.0 are demonstrated in this section for three full scale applications. The first two cases highlight usage of the conjugate gradient method to obtain the total acoustic pressure at a field of observers bisecting 1) a typical high bypass ratio nacelle, and 2) a commercial transport (fuselage, wings, nacelles); the last case features usage of the SLFMM to simulate the scattered acoustic pressure field at a plane bisecting the futuristic SAX- $40^{10}$ configuration. All calculations presented here were performed on a single processor of a Beowulf cluster with sixteen Itanium 2, $1.4 \mathrm{GHz}$ processors and 32 GB of shared RAM.

\section{A. High Bypass Ratio Nacelle}

Because of the large surface area of modern high bypass ratio engine nacelles, predictions using FSC v2.0 are limited to frequencies up to $1.5 \mathrm{x}$ BPF using current single-processor computer technology. In order to benchmark the CGM implementation with available resources, a nacelle surface similar to the GE-90 was discretized for $1.5 \mathrm{x}$ $\mathrm{BPF}$ and used with an excitation frequency of $3 \mathrm{x}$ BPF. The incident acoustic field was generated using spinning monopoles of unit strength placed at the rotor blade tips. The number of monopoles was chosen so as to excite the lowest radiating circumferential mode for this frequency $(\mathrm{m}=12)$. For simplicity, forward Mach number is zero. Contours of total acoustic pressure obtained with FSC v2.0 are presented in Figure 2a for a plane bisecting the nacelle and Fig. $2 \mathrm{~b}$ for a plane located $0.05 \mathrm{~m}$ forward of the rotor disc. Contours of sound pressure level (SPL) are given in Figure 3. Although the computational mesh contains only a fraction of the collocation points (25\%) and equivalent sources $(25 \%)$ necessary for proper wave resolution at $3 \times \mathrm{BPF}$, note from the figures that the correct trend is simulated - propagation of multiple radial modes through the nacelle openings.

CGM validation with a null initial guess against known FSC v2.0 results indicated that the method is robust and converges rapidly to the correct solution for single component configurations at moderate frequencies. At higher frequencies, convergence is improved using a non-zero initial guess obtained with a subset of the required equivalent sources. A coarser mesh with point density $1 / 4$ of fine was used for this exercise. Instantaneous total acoustic pressure contours for the initial guess and final solution are given in Figures $4 \mathrm{a}$ and $4 \mathrm{~b}$, respectively. Note that the CGM fine grid solution is virtually identical to that obtained with the direct method, shown in Fig. 2 a. Statistics for the different aspects of this application are presented in Table 1. Note that computational time was reduced by a factor of 2.5 .

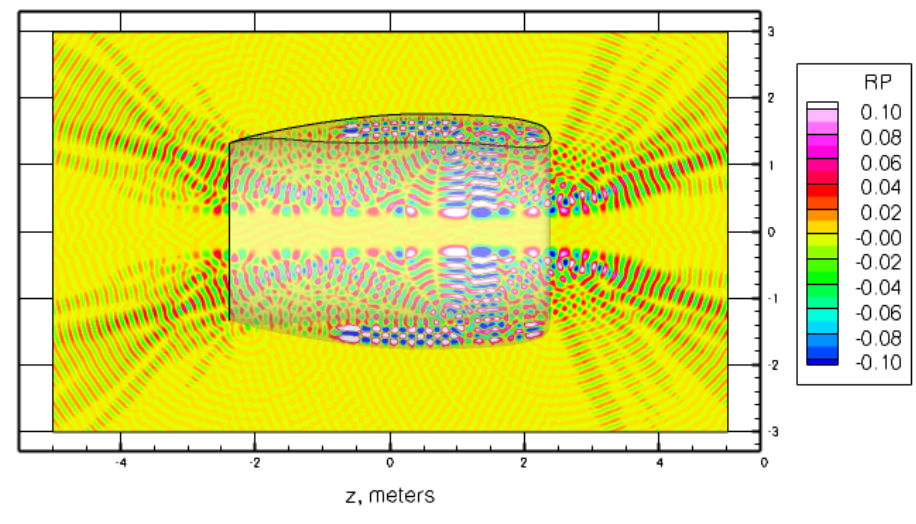

(a) Bisecting plane

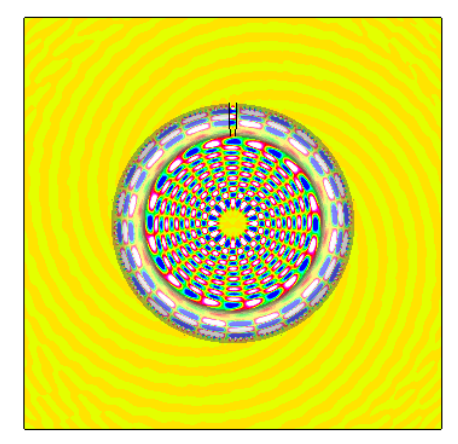

(b) Plane $0.05 \mathrm{~m}$ forward of rotor disc

Figure 2. Instantaneous Re (p') contours, in Pa, for a full scale GE-90 like nacelle; $f=3$ x BPF, $M=0.0, m=12$. FSC $\mathbf{v} 2.0$ results. 


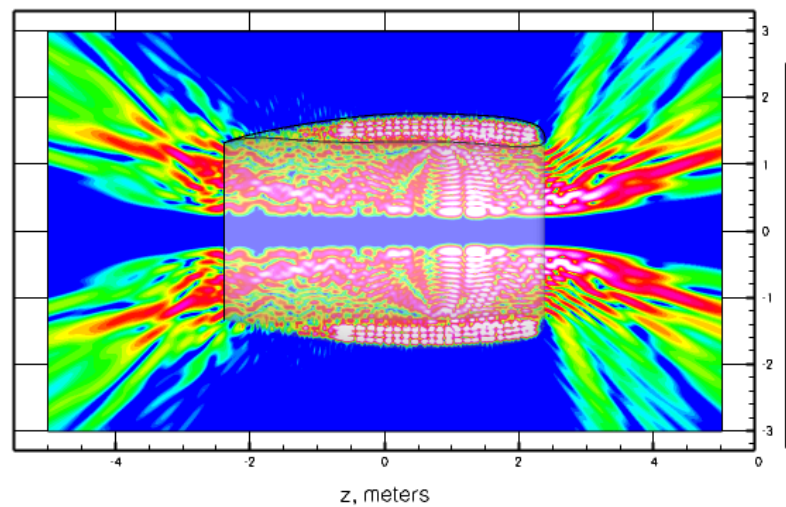

(a) Bisecting plane

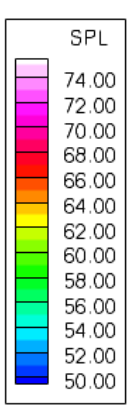

(b) Plane $0.05 \mathrm{~m}$ forward of rotor disc

Figure 3. Instantaneous SPL contours, in $\mathrm{dB}$, for a full scale GE-90 like nacelle; $f=3 \times \mathrm{BPF}, \mathrm{M}=\mathbf{0 . 0}, \mathrm{m}=12$. FSC v2.0 results.

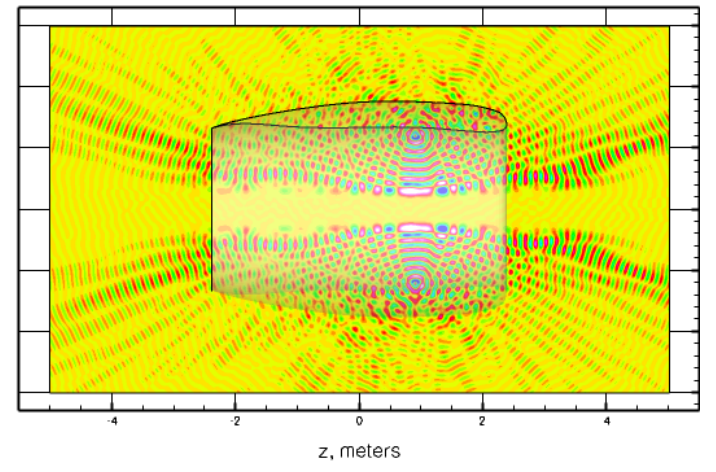

(a) FSC-CGM, initial guess

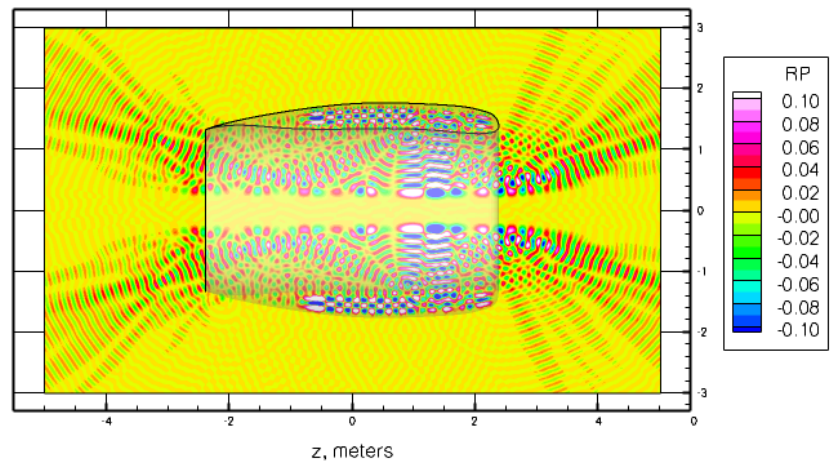

(b) FSC-CGM, fine grid

Figure 4. Instantaneous $R e\left(p^{\prime}\right)$ contours, in Pa, for a full scale GE-90 like nacelle; $f=3 \times 3$ BP, $M=0.0$, $\mathbf{m}=12$. FSC-CGM results.

Table 1. Statistics for full scale nacelle, $f=3$ x BPF (with 1.5 x BPF grid).

\begin{tabular}{|c|c|c|l|c|}
\hline Parameter & Initial guess & Fine grid & Cummulative & $\begin{array}{c}\text { Improvement } \\
\text { over DM }\end{array}$ \\
\hline No. iterations & 74 & 1198 & & 2.53 \\
\hline Solution time, $\mathrm{s}$ & 137 & 9888 & 10052 & \\
\hline $\begin{array}{c}\text { Direct method (DM) } \\
\text { solution time, } \mathrm{s}\end{array}$ & \multicolumn{3}{|c|}{25413} \\
\hline
\end{tabular}

\section{B. Commercial Transport}

The most challenging application for the FSC with the SLFMM/CGM methodology is high frequency predictions for large scale commercial transports. Current FSC practice is limited to excitation frequencies of about $0.4 \times$ BPF of engine fan noise. In order to test the CGM implementation on an arbitrary, multi-component geometry with available computer resources, a configuration similar to the Boeing 777 (wings, fuselage and nacelles) 
discretized for 0.4 x BPF was used with an excitation frequency of 1 x BPF. Forward velocity was zero. The incident acoustic field was generated using the engine noise feedback option, which allows the user to input a preexisting nacelle-only solution into a current FSC run. A nacelle similar to the GE-90 was used for this exercise. Because all axial modes are cut-off for $1 \mathrm{x}$ BPF, stationary monopoles at the nacelle centers were used to generate the input solution. For simplicity, forward velocity was zero.

Contours of total acoustic pressure obtained with FSC v2.0 are presented in Figure 5 for a plane bisecting the configuration; SPL contours are given in Figure 6. Shielding of engine noise by the wings and fuselage is apparent, although the computational mesh contains only a fraction (about 20\%) of the required collocation points and equivalent sources associated with the frequency under consideration. Results using the CGM algorithm with a nonzero initial guess obtained with a $1 / 4$ density mesh are presented in Figure 7 . Note that the fine mesh solution is very similar to that obtained with the direct method (see Fig. 5), even though the iteration limit was reached. Statistics for the different aspects of this application are presented in Table 2. For this case, computational time was reduced by a factor of 1.38 .

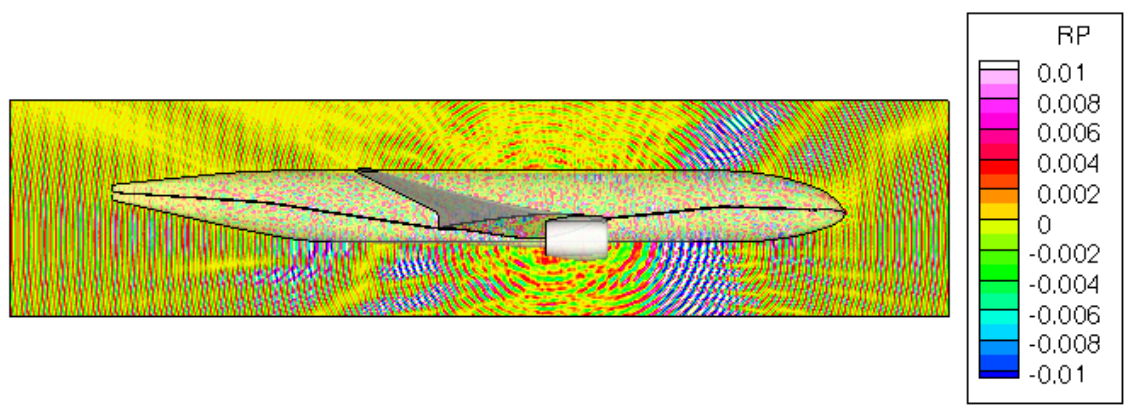

Figure 5. Instantaneous contours of Re (p') for a full scale commercial transport; $f=1 \times$ BPF (with $0.4 x$ $B P F$ grid), $M=0.0$. FSC v2.0 results with engine noise feedback.
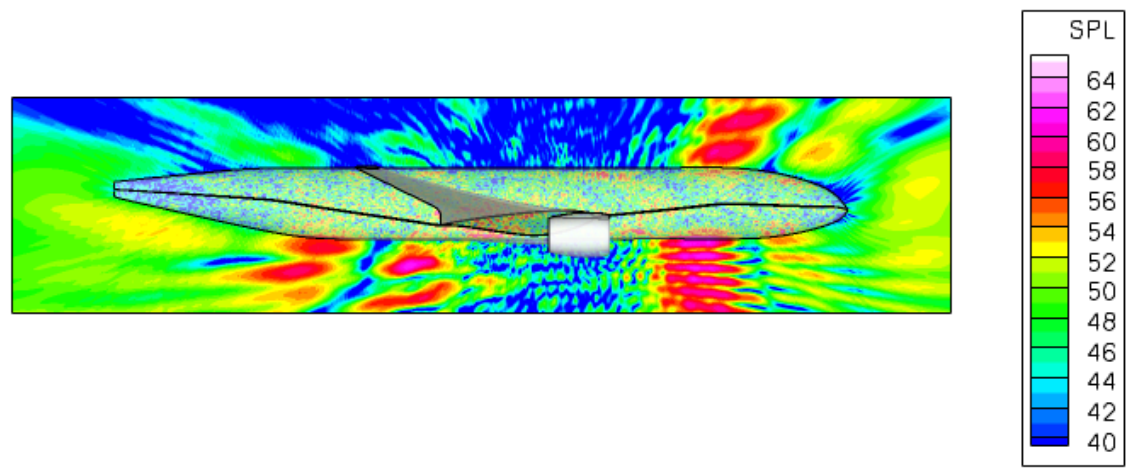

Figure 6. Instantaneous SPL contours for a full scale commercial transport; $f=1 \times$ BPF (with $0.4 \times$ BPF grid), $M=0.0$. FSC v2.0 results with engine noise feedback.

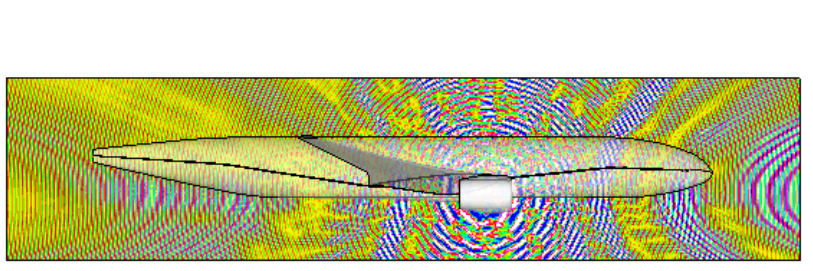

(a) FSC-CGM, initial guess
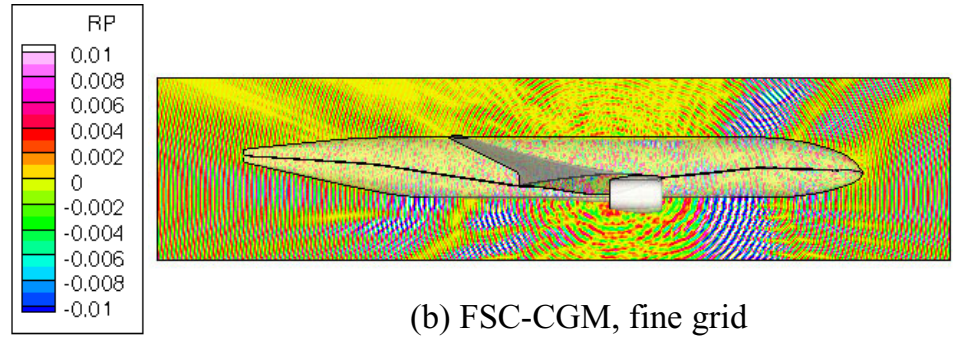

(b) FSC-CGM, fine grid

Figure 7. Instantaneous Re (p') contours for a full scale commercial transport; $f=1$ x BPF (with 0.4 x BPF grid), $M=0.0$. FSC - CGM results with engine noise feedback. 
Table 2. Statistics for full scale commercial transport, $f=1 \times$ BPF (with $0.4 \times$ BPF grid).

\begin{tabular}{|c|c|c|c|c|}
\hline Parameter & Initial guess & Fine grid & Cummulative & $\begin{array}{c}\text { Improvement } \\
\text { over DM }\end{array}$ \\
\hline No. iterations & 466 & $2500^{\mathrm{a}}$ & & 1.38 \\
\hline Solution time, s & 1090 & 26188 & 27370 & 37887 \\
\hline $\begin{array}{c}\text { Direct method (DM) } \\
\text { solution time, } \mathrm{s}\end{array}$ & \multicolumn{2}{|c|}{} \\
\hline
\end{tabular}

a. Iteration limit reached.

\section{Blended Wing Body Concept (SAX-40)}

The configuration considered in this exercise resulted from the Silent Aircraft Initiative, a collaborative effort among industry, academia and government aimed at determining the aerodynamic and performance characteristics of a conceptual vehicle using noise reduction as a prime design variable. The final design, known as SAX-40, incorporates three boundary layer ingesting engine clusters. A variation of the design, with three pod-type nacelles placed above the fuselage, was used here.

The incident acoustic field, generated using the engine noise feedback option, is that due to spinning monopoles of circumferential mode order zero $(\mathrm{m}=0)$ located within the nacelles at the fan face. Available resources limited the FSC v2.0 simulations to an excitation frequency of $400 \mathrm{~Hz}$ using a subset computational mesh corresponding to $200 \mathrm{~Hz}$. Contours of SPL (including contributions from all three engines) at a plane bisecting the configuration are presented in Figure 8. The plane contains 255 x 510 points. Note from the figure that, although the acoustic waves are not properly resolved for the frequency of interest, shielding of fan inlet noise by the body is apparent. Contours of scattered acoustic pressure at the same observer field are given in Figure 9a. The SLFMM implementation, in conjunction with equivalent source strengths previously calculated with FSC v2.0, was used to simulate the scattered acoustic field presented in Figure 9b. Observe that the fields are virtually identical. Statistics for the different aspects of this test are included in Table 3. Computational time was reduced by a factor of 3.78 .
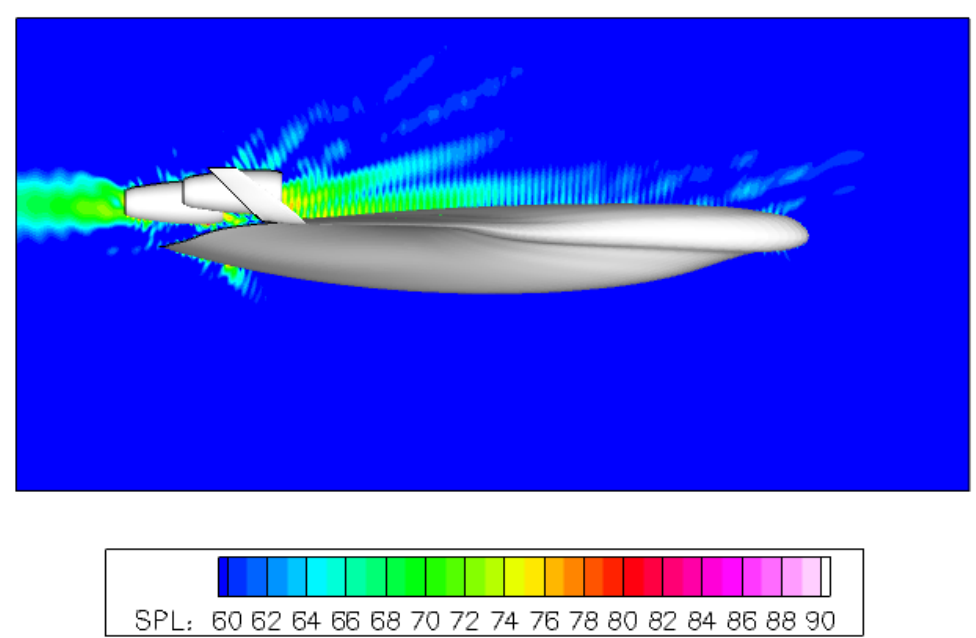

Figure 9. Instantaneous SPL contours, in $\mathrm{dB}$, for the SAX-40 conceptual design with pod nacelles; $\mathrm{f}=400 \mathrm{~Hz}$ (with $200 \mathrm{~Hz}$ grid), $M=0.0, \mathrm{~m}=0$. FSC v2.0 results. 


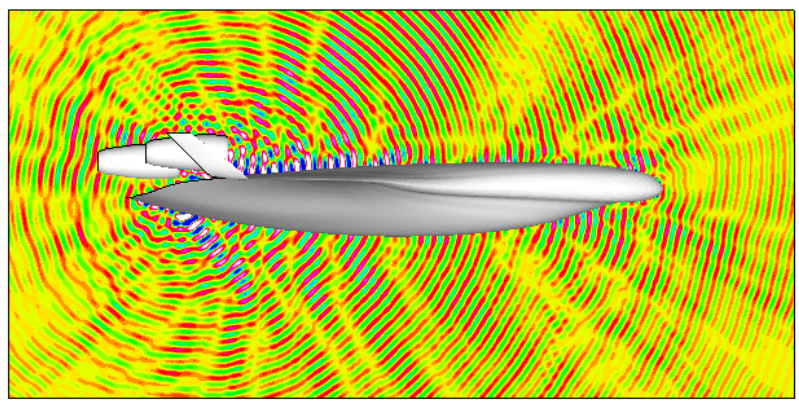

(a) FSC v2.0

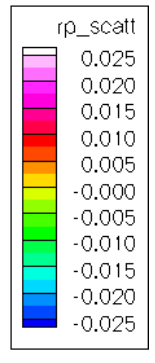

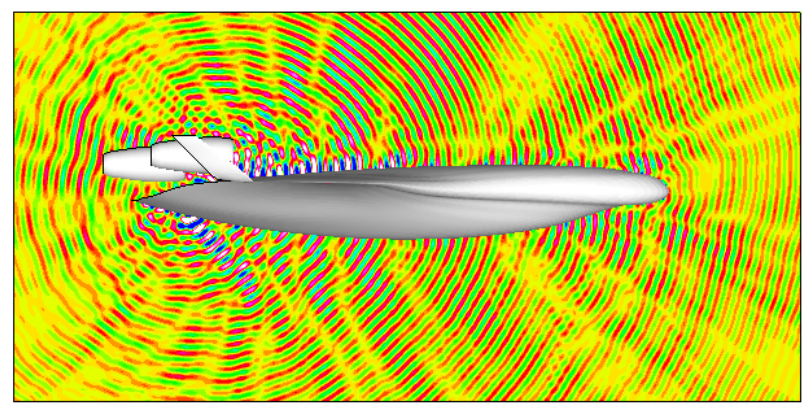

(b) FSC - SLFMM

Figure 10. Instantaneous $\mathrm{Re}\left(p_{\text {scat }}^{\prime}\right)$ contours, in Pa, for the SAX-40 design with pod nacelles; $f=400 \mathrm{~Hz}$ (with $200 \mathrm{~Hz}$ grid), $\mathrm{M}=\mathbf{0 . 0}, \mathbf{m}=\mathbf{0}$.

Table 3. Statistics for SAX-40 configuration, $f=400 \mathrm{~Hz}$ (with $200 \mathrm{~Hz}$ grid).

\begin{tabular}{|l|c|c|}
\hline \multicolumn{1}{|c|}{ Parameter } & Value & $\begin{array}{c}\text { Improvement over } \\
\text { DM }\end{array}$ \\
\hline Terms in spherical expansion & 23 & \\
\hline Non-empty boxes & 325 & \\
\hline Average points per box & 400 & \\
\hline Box size, $\mathrm{m}$ & 2.165 & 3.78 \\
\hline Storage (relative to DM), \% & 3.5 & \\
\hline Time, $\mathrm{s}$ & 2399 & \\
\hline Direct method time, $\mathrm{s}$ & & \multicolumn{2}{|c|}{9072} \\
\hline
\end{tabular}

\section{Concluding Remarks}

The NASA Fast Scattering Code is a versatile analysis tool developed to conduct aeroacoustic noise reduction studies. The program employs the equivalent source method to solve a three-dimensional exterior Helmholtz boundary value problem. FSC v2.0 uses dense matrix solution techniques that are memory intensive and time consuming, limiting applications to problems of moderate size. Calculations are dominated by the matrix-matrix multiplication required by the pre-conditioning step of the solution process. Application of the single-level fast multipole method and sparse linear algebra techniques eliminates the matrix-matrix multiply and has led to an orderof-magnitude reduction in computational resource requirements, thereby doubling the excitation frequency achievable for a given configuration. In the program FSC v3.0, iterative conjugate gradient methods have been coupled to fast matrix-vector multiplication algorithms based on the SLFMM. At the end of the solution process, the SLFMM is deployed again for the rapid summation of the acoustic field. Sample calculations for several configurations of importance have been presented to demonstrate the SLFMM analysis and speed-up gains.

Further computational advances are possible with the SLFMM technology as presented here, and will be addressed by the authors in future releases of the FSC. First, $O\left(q^{3}\right)$ translation algorithms exist in the literature for faster evaluation of the regular expansion coefficients in Eq. (11) which is an $O\left(q^{4}\right)$ algorithm. Second, multi-level fast multipole methods (MLFMM) offer an additional degree of computational time reduction. By repeatedly subdividing the computational boxes into smaller boxes, analysis shows that the complexity of the SLFMM can be 
reduced from $O\left(N^{4 / 3}\right)$ to $O(N \log N)$ by application of the MLFMM algorithms ${ }^{8}$. These two research aspects account for marginal gains in the FSC performance. More profound computational advantages can be obtained through the use of multi-processor computer technology. Step 2 in the evaluation of the SLFMM algorithm involves the calculation of the regular expansion for all points in each receiver box. Since receiver box calculations are independent of each other, it is possible to distribute the computational work for the boxes over an array of processors reducing the work load by a multiplicative factor approaching the number of processors. Incorporation of these additional research methods is expected to be completed by early 2009 .

\section{Acknowledgments}

Development of the FSC v3.0 is sponsored by the Aeroacoustics Branch of the NASA LaRC under NRA contract No. NNL07AA24C.

\section{References}

${ }^{1}$ Posey, J. W., Tinetti, A. F., and Dunn, M. H., "The low Noise Potential of Distributed Propulsion on a Catamaran Aircraft," AIAA paper 2006-2622, May 2006.

${ }^{2}$ Farassat, F., Casper, J. H., Tinetti, A. F., and Dunn, M. H., “Airframe Noise Prediction by Acoustic Analogy: Revisited," AIAA paper 2006-2564, May 2006.

${ }^{3}$ Reimann, C. A., Tinetti, A. F., and Dunn, M. H., "Noise Scattering by the Blended Wing Body Airplane: Measurements and Predicition," AIAA paper 2006-2474, May 2006.

${ }^{4}$ Reimann, C. A., Tinetti, A. F., and Dunn, M. H., "Engine Liner Optimization Using the Fast Scattering Code," AIAA paper 2007-3494, May 2007.

${ }^{5}$ Lee, S., Erwin, J. P., and Brentner, K., "Acoustic Scattering of Rotorcraft Noise," presented at the American Helicopter Society $64^{\text {th }}$ Annual Forum, Montreal, Canada, April 29 - May 1, 2008.

${ }^{6}$ Dunn, M. H., and Tinetti, A. F., “Aeroacoustic Scattering Via the Equivalent Source Method,” AIAA 2004-2937, May 2004.

${ }^{7}$ Tinetti, A. F., Dunn, M. H., and Pope, D. S., "Fast Scattering Code (FSC) User's Manual, Version 2.0," NASA CR 2006214510, October 2006.

${ }^{8}$ Gumerov, N. A., and Duraiswami, R., Fast Multipole Methods for the Helmholtz Equation in Three Dimensions, first edition, Oxford, UK, Elsevier, 2004.

${ }^{9}$ Bjorck, A., Numerical Methods for Least Squares Problems, first edition, Philadelphia, SIAM, 1996.

${ }^{10}$ Hileman, J. I., Spakovsky, Z. S., Drela, M., and Sargeant, M. A., “Airframe Design for Silent Aircraft,” AIAA paper $2007-$ 453, January 2007. 